\title{
An approximation process of Kantorovich type
}

\author{
Octavian Agratini
}


Mathematical Notes, Miskolc, Vol. 2., No. 1., (2001), pp. 3-10

\title{
AN APPROXIMATION PROCESS OF KANTOROVICH TYPE
}

\author{
Octavian Agratini \\ Faculty of Mathematics and Computer Science, Babeş-Bolyai University \\ Kogălniceanu 1, 3400 Cluj-Napoca, Romania \\ agratini@math.ubbcluj.ro
}

[Received December 11, 2000]

\begin{abstract}
The paper is devoted to the study of an approximation process on an unbounded interval representing an integral form in Kantorovich sense of K. Balazs operators. We establish the degree of approximation in some function spaces pointing out the relationship between the local smoothness of functions and the local approximation. By using the modulus of variation, the approximation property in discontinuity points is also examined.
\end{abstract}

Mathematical Subject Classification: 41A36

Keywords: Kantorovich-type operator, Bohman-Korovkin theorem, modulus of smoothness of first order, modulus of variation, local Lip $\alpha(0<\alpha \leq 1)$

\section{Introduction}

In [1] Katalin Balázs introduced and considered some approximation properties of the discrete linear operators defined by

$$
\left(R_{n} f\right)(x)=\frac{1}{\left(1+a_{n} x\right)^{n}} \sum_{k=0}^{n}\left(\begin{array}{l}
n \\
k
\end{array}\right)\left(a_{n} x\right)^{k} f\left(\frac{k}{b_{n}}\right), \quad x \geq 0, \quad n \in \mathbb{N},
$$

where $a_{n}$ and $b_{n}$ are suitably chosen positive numbers, independent of $x$, and the function $f$ belongs to $[0, \infty)$. In the particular case

$$
a_{n}=n^{\beta-1}, \quad b_{n}=n^{\beta}, \quad n \in \mathbb{N}, \quad 0<\beta<1,
$$

the above operators will be denoted by $R_{n}^{[\beta]}$.

In [1], for the operators defined by (1.1) a Voronovskaja type formula was given under the hypothesis that $a_{n}=b_{n} / n \rightarrow 0$ and $\sqrt{n} / b_{n} \rightarrow 0(n \rightarrow \infty)$. In the same paper, for $R_{n}^{[2 / 3]}$ operators convergence theorems and the convergence of their derivatives to the derivative of the function were proved.

In [2], for $R_{n}^{[\beta]}$ operators, $0<\beta \leq 2 / 3$, weighted estimates were given and certain questions of the uniform convergence of $R_{n} f$ on $(0, \infty)$ were also developed.

In his extensive paper [3], V. Totik settled the saturation properties of $R_{n}^{[\beta]} f$, $0<\beta<1$, and proved a general convergence theorem for $R_{n}$-like rational functions. 
In 1989 , for $R_{n}^{[\beta]}, 0<\beta<1$, Biancamaria Della Vecchia [4] obtained some preservation properties and weak asymptotic relations generalizing the result of Totik. Regarding the same operators, the most recent paper is due to Ulrich Abel and B. Della Vecchia [5] who obtained the complete asymptotic expansion for $R_{n}^{[\beta]} f$ as $n$ tends to infinity.

The aim of this paper is to investigate a generalization of $R_{n}$ operators in Kantorovich sense. In the next section we present the integral extension and in the third part some approximation properties are revealed, establishing the rate of pointwise convergence for various classes of functions such as continuous functions, local Lipo $(0<\alpha \leq 1)$ functions and locally bounded functions having the one-sided limits at a given $x>0$. In our investigation we use the modulus of smoothness of first order and the Chanturiya modulus of variation.

\section{An integral extension}

Throughout the paper $e_{n}$ represents the monomial $e_{n}(x)=x^{n}$, where $n$ belongs to $\mathbb{N}_{0}:=\mathbb{N} \cup\{0\}$.

Let us denote by $M_{l o c}([0, \infty))$ the class of all functions measurable on $[0, \infty)$ and bounded on every compact subinterval of $[0, \infty)$.

Concerning the operators $R_{n}, n \in \mathbb{N}$, defined by (1) we consider that $b_{n}=n a_{n}>0$ holds for every $n \in \mathbb{N}$, a requirement already formulated in some previous papers. Now we modify these operators into integral form operators by replacing $f\left(k / b_{n}\right)$ with an integral mean of $f(x)$ over a small interval named $I_{n, k}=\left[\frac{k}{n a_{n}}, \frac{k+1}{n a_{n}}\right]$, as follows

$$
\left(K_{n} f\right)(x)=n a_{n} \sum_{k=0}^{n} r_{n, k}(x) \int_{I_{n, k}} f(t) d t, \quad x \geq 0, \quad n \in \mathbb{N},
$$

where $f \in M_{l o c}([0, \infty))$ and

$$
r_{n, k}(x)=\left(\begin{array}{l}
n \\
k
\end{array}\right)\left(a_{n} x\right)^{k}\left(1+a_{n} x\right)^{-n}, \quad 0 \leq k \leq n .
$$

We can see that $K_{n}$ is a linear positive operator and it is stable in the Fejérsense, i.e. $\inf _{t \in I_{n}} f(t) \leq\left(K_{n} f\right)(x) \leq \sup _{t \in I_{n}} f(t), x \geq 0$, where $I_{n}=\left[0, \frac{n+1}{n a_{n}}\right]$. Since $\sum_{k=0}^{n} r_{n, k}(x)=1$, we obtain

$$
\left(K_{n} e_{0}\right)(x)=1, \quad x \geq 0, \quad n \in \mathbb{N},
$$

in other words, $K_{n}$ reproduces the constants.

Remarks. (i) Like any linear operators of discrete type, $R_{n}, n \in \mathbb{N}$, are not suitable for approximation to discontinuous functions of a general type because they 
use information about $f$ which comes from some certain points in the domain of $f$. On the other hand, the operators $K_{n}, n \in \mathbb{N}$, are constructed based on integral means of $f$ over small intervals, so we may obtain better results in approximating the discontinuous functions.

(ii) We can denote more explicitly $K_{n} f$ by $K_{n}\left(a_{n}, \mathbf{r}_{n}, f ; \cdot\right)$ where $\mathbf{r}_{n}=\left(r_{n, k}\right)_{0 \leq k \leq n}$. We consider the function $\sigma:[0,1) \rightarrow[0, \infty), \sigma(t)=t /(1-t)$ and $\widetilde{\mathbf{r}}_{n}=\left(\widetilde{r}_{n, k}\right)_{0 \leq k \leq n}$ where $\widetilde{r}_{n, k}(x):=\left(r_{n, k} \circ \sigma\right)(x), 0 \leq x<1$, and $\widetilde{r}_{n, k}(1):=\delta_{n, k}$ (Kronecker symbol). If we choose $a_{n}=1$, then by using (2.1) we obtain

$$
K_{n+1}\left(1, \widetilde{\mathbf{r}}_{n}, f ; x\right)=(n+1) \sum_{k=0}^{n}\left(\begin{array}{l}
n \\
k
\end{array}\right) x^{k}(1-x)^{n-k} \int_{\frac{k}{n+1}}^{\frac{k+1}{n+1}} f(t) d t
$$

which represents the $n-t h$ Kantorovich operator, a well-known approximation process in spaces of integrable functions. For more details we refer e.g. to the monograph $[6$; 5.3.7.].

(iii) We can write the operator $K_{n}$ as a singular integral of the type

$$
\left(K_{n} f\right)(x)=\int_{0}^{\infty} k_{n}(x, t) f(t) d t, \quad x \geq 0,
$$

where $k_{n}$ is a non-negative kernel defined as follows

$$
\begin{aligned}
& k_{n}(x, t)=n a_{n} r_{n, k}(x) \text { for } x \geq 0, k /\left(n a_{n}\right)<t \leq(k+1) /\left(n a_{n}\right) \text { and } \\
& k_{n}(x, t)=0 \text { for } x \geq 0, t \in\{0\} \cup\left(\frac{n+1}{n a_{n}}, \infty\right) .
\end{aligned}
$$

Using (2.3) it is obvious that our kernel satisfies $\int_{0}^{\infty} k_{n}(x, t) d t=1$.

Example. We choose $a_{n}=n^{\beta-1}, n \in \mathbb{N}$, where $0<\beta<1$ is fixed. The operator defined by (2.1) becomes the integral analogue (named $K_{n}^{[\beta]}$ ) of $R_{n}^{[\beta]}$ defined by (1.1) and (1.2). More precisely, we have

$$
\left(K_{n}^{[\beta]} f\right)(x)=\frac{n^{\beta}}{\left(1+n^{\beta-1} x\right)^{n}} \sum_{k=0}^{n}\left(\begin{array}{l}
n \\
k
\end{array}\right)\left(n^{\beta-1} x\right)^{k} \int_{k / n^{\beta}}^{(k+1) / n^{\beta}} f(t) d t, \quad x \geq 0 .
$$

\section{Some approximation properties of the operators $K_{n}$}

Setting $T_{i}(y)=\sum_{k=0}^{n} k^{i}\left(\begin{array}{l}n \\ k\end{array}\right) y^{k}, i \in \mathbb{N}_{0}$, the following elementary identities hold true

$$
T_{1}(y)=n y(1+y)^{n-1} \quad \text { and } \quad T_{2}(y)=n(n-1) y^{2}(1+y)^{n-2}+T_{1}(y), \quad n \geq 1 .
$$

Taking into account (2.1) and (3.1), we have

$$
\left(K_{n} e_{1}\right)(x)=\frac{1}{2 n a_{n}}\left(\frac{2 T_{1}\left(a_{n} x\right)}{\left(1+a_{n} x\right)^{n}}+1\right)=\frac{x}{1+a_{n} x}+\frac{1}{2 n a_{n}},
$$


and

$$
\begin{aligned}
\left(K_{n} e_{2}\right)(x) & =\frac{1}{3 n^{2} a_{n}^{2}}\left(\frac{3 T_{2}\left(a_{n} x\right)+3 T_{1}\left(a_{n} x\right)}{\left(1+a_{n} x\right)^{n}}+1\right)= \\
& =\frac{n-1}{n} \frac{x^{2}}{\left(1+a_{n} x\right)^{2}}+\frac{2 x}{n a_{n}\left(1+a_{n} x\right)}+\frac{1}{3 n^{2} a_{n}^{2}},
\end{aligned}
$$

respectively.

Further, we consider $\mathcal{M}_{n, s}(x):=\left(K_{n}\left(e_{1}-x e_{0}\right)^{s}\right)(x)$

${ }_{n, s}(x):=\left(K_{n}\left(e_{1}-x e_{0}\right)^{s}\right)(x), s \in \mathbf{N}$, representing the central moment of order $s$ for $K_{n}$ operator. Taking advantage of the identities regarding $K_{n} e_{j}, j \in\{0,1,2\}$, we obtain the following result.

Lemma 1. If the operators $K_{n}, n \in \mathbb{N}$, are defined by (2.1), then we get

i) ${ }_{n, 1}(x)=-\frac{a_{n} x}{1+a_{n} x}+\frac{1}{2 n a_{n}}$,

ii) ${ }_{n, 2}(x)=\frac{n a_{n}^{2} x^{2}-1}{n\left(a_{n} x+1\right)^{2}} x^{2}+\frac{1-a_{n} x}{n a_{n}\left(1+a_{n} x\right)} x+\frac{1}{3 n^{2} a_{n}^{2}}$.

By using these relations we can state

Lemma 2. Let $K_{n}, n \in \mathbb{N}$, be defined by (2.1) such that $a_{n} \rightarrow 0$, na $a_{n} \rightarrow \infty$ as $n \rightarrow \infty$. Then we have

$$
\lim _{n \rightarrow \infty} n, j(x)=0, \quad \text { for every } \quad x \geq 0 \quad \text { and } \quad j \in\{1,2\}
$$

Theorem 1. Let $K_{n}, n \in \mathbb{N}$, be defined by (2.1). If $a_{n} \rightarrow 0, n a_{n} \rightarrow \infty$ as $n \rightarrow \infty$, then for every $f \in C([0, \infty))$ one has

$$
\lim _{n \rightarrow \infty} K_{n} f=f \quad \text { uniformly on any compact } K \subset[0, \infty) \text {. }
$$

Proof. Our assertion results directly from the well-known theorem of BohmanKorovkin, relation (2.3) and Lemma 2 as well.

We are going to study the degree of approximation in terms of $\omega_{1}$, the modulus of smoothness of first order, defined as

$$
\omega_{1}(f ; s)=\sup \{|f(x)-f(y)|: x \geq 0, y \geq 0,|x-y| \leq s\} .
$$

Theorem 2. If the operators $K_{n}, n \in \mathbb{N}$, are defined by (2.1), then for each $x \geq 0$ the following inequality

$$
\left|\left(K_{n} f\right)(x)-f(x)\right| \leq 2 \omega_{1}\left(f ; \delta_{n, x}\right)
$$

holds, where

$$
\delta_{n, x}=\left(\max \left\{a_{n} x, \frac{1}{n}\right\} x^{2}+\frac{x}{n a_{n}}+\frac{1}{3 n^{2} a_{n}^{2}}\right)^{1 / 2}
$$


and $f \in M_{l o c}([0, \infty))$.

Proof. Since (2.3) holds and $K_{n}$ is a positive operator, we can write

$$
\left|\left(K_{n} f\right)(x)-f(x)\right| \leq a n_{n} \sum_{k=0}^{n} r_{n, k}(x) \int_{k /\left(n a_{n}\right)}^{(k+1) /\left(n a_{n}\right)}|f(t)-f(x)| d t .
$$

On the other hand, the definition of the modulus of smoothness $\omega_{1}(f ; \cdot)$ implies

$$
|f(t)-f(x)| \leq \omega_{1}(f ;|t-x|) \leq\left(1+\delta^{-2}(t-x)^{2}\right) \omega_{1}(f ; \delta), \quad \delta>0 .
$$

We have also used both the fact that $\omega_{1}(f ; \cdot)$ is an increasing function and the property $\omega_{1}(f ; \lambda \delta) \leq\left(1+\lambda^{2}\right) \omega_{1}(f ; \delta)$ for every $\lambda>0$ and $\delta>0$.

Substituting (3.3) in (3.2) and taking into account (2.3) it results

$$
\left|\left(K_{n} f\right)(x)-f(x)\right| \leq\left(1+\delta_{n, 2}^{-2}(x)\right) \omega_{1}(f ; \delta), \quad \delta>0 .
$$

At this step we consider $\delta={ }_{n, 2}^{1 / 2}(x)$. If we use Lemma 1 (ii) and the following relations

$$
\left|\frac{a_{n} x-1}{a_{n} x+1}\right| \leq 1, \quad-\frac{1}{n} \leq \frac{n a_{n}^{2} x^{2}-1}{n\left(a_{n} x+1\right)^{2}}<a_{n} x, \quad n \in \mathbb{N}, \quad x \geq 0,
$$

then we deduce that ${ }_{n, 2}(x) \leq \max \left\{a_{n} x, \frac{1}{n}\right\} x^{2}+\frac{x}{n a_{n}}+\frac{1}{3 n^{2} a_{n}^{2}}:=\delta_{n, x}^{2}$. The desired result follows.

Corollary. If the operators $K_{n}^{[\beta]}, n \in \mathbb{N}, 0<\beta<1$, are defined by (6), then for each $x \geq 0$ and $\lambda>0$ we have

$$
\left|\left(K_{n}^{[\beta]} f\right)(x)-f(x)\right| \leq\left(1+n^{-\lambda}\right) \omega_{1}\left(f ; \theta_{n, x}\right),
$$

where $\theta_{n, x}=\left(\max \left\{n^{\lambda+\beta-1} x, n^{\lambda-1}\right\} x^{2}+n^{\lambda-\beta} x+n^{\lambda-2 \beta}\right)^{1 / 2}$ and $f \in M_{l o c}([0, \infty))$.

Proof. We apply Theorem 2 for the special case $a_{n}=n^{\beta-1}$. Also, in (3.4) we choose $\delta=n_{n, 2}^{\lambda / 2}{ }_{n, 2}^{1 / 2}(x)$ and further $\theta_{n, x}=n^{\lambda} \delta_{n, x}^{2}$. The proof is complete.

Remark. If the real number $\lambda$ satisfies $0<\lambda<\min \{1-\beta, \beta\}$, then $\theta_{n, x} \rightarrow 0$ as $n \rightarrow \infty$.

Theorem 3. Let $K_{n}$ be given by (2.1), $0<\alpha \leq 1$ and $E$ be any subset of $[0, \infty)$. If $f$ is local Lipa on E, i.e.,

$$
|f(x)-f(y)| \leq M_{f}|x-y|^{\alpha},(\forall)(x, y) \in[0, \infty) \times E,
$$

then we have

$$
\left|\left(K_{n} f\right)(x)-f(x)\right| \leq M_{f}\left(\varepsilon_{n}(x)+\frac{1}{\left(n a_{n}\right)^{\alpha}}+2 d^{\alpha}(x, E)\right),
$$


where $M_{f}$ is a constant depending only on $\alpha$ and $f$,

$$
\varepsilon_{n}(x)=\left(\max \left\{a_{n} x, \frac{1}{n}\right\} x^{2}\right)^{\alpha / 2}+\left(\frac{x}{n a_{n}}\right)^{\alpha / 2}
$$

and $d(x, E)$ is the distance between $x$ and $E$ defined as $d(x, E)=\inf \{|x-y|: y \in E\}$.

Proof. It is clear that (3.6) holds for any $x \geq 0$ and $y \in \bar{E}$, the closure in $\mathbb{R}$ of the set $E$. Let $\left(x, x_{0}\right) \in[0, \infty) \times \bar{E}$ be such that $\left|x-x_{0}\right|=d(x, E)$. At this point we can write $|f-f(x)| \leq\left|f-f\left(x_{0}\right)\right|+\left|f\left(x_{0}\right)-f(x)\right|$ and applying the linear and positive operator $K_{n}$ we have

$$
\begin{aligned}
\left|\left(K_{n} f\right)(x)-f(x)\right| & \leq K_{n}\left(\left|f-f\left(x_{0}\right)\right|, x\right)+\left|f(x)-f\left(x_{0}\right)\right| \leq \\
& \leq K_{n}\left(M_{f}\left|e_{1}-x_{0} e_{0}\right|^{\alpha}, x\right)+M_{f}\left|x-x_{0}\right|^{\alpha} \leq \\
& \leq M_{f}\left(K_{n}\left(\left|e_{1}-x e_{0}\right|^{\alpha}, x\right)+2\left|x-x_{0}\right|^{\alpha}\right) \leq \\
& \leq M_{f}\left(\begin{array}{c}
\alpha / 2 \\
n, 2
\end{array}(x)+2\left|x-x_{0}\right|^{\alpha}\right) .
\end{aligned}
$$

We used the classical inequalities $\left|t-x_{0}\right|^{\alpha} \leq\left(|t-x|+\left|x-x_{0}\right|\right)^{\alpha} \leq|t-x|^{\alpha}+\left|x-x_{0}\right|^{\alpha}$, and $K_{n}\left(h^{\alpha}, x\right) \leq\left(K_{n}\left(h^{2}, x\right)\right)^{\alpha / 2}, 0<\alpha \leq 1$. The latter is a direct result of Hölder's inequality and relation (2.3).

On the other hand, Lemma 1 and relation (3.5) lead us to the following inequality

$$
{ }_{n, 2}^{\alpha / 2}(x) \leq\left(\max \left\{a_{n} x, \frac{1}{n}\right\}\right)^{\alpha / 2} x^{\alpha}+\left(\frac{x}{n a_{n}}\right)^{\alpha / 2}+\left(\frac{1}{n a_{n}}\right)^{\alpha}=\varepsilon_{n}(x)+\frac{1}{\left(n a_{n}\right)^{\alpha}} .
$$

Returning to (3.8), the proof is complete.

In particular, for $E=[0, \infty)$ in $(3.7)$ the term $d(x, E)$ vanishes.

In the final part of this section we present a general quantitative estimate for the rate of pointwise convergence of $\left(K_{n} f\right)(x), x>0$, for the functions $f \in M_{l o c}([0, \infty))$ with the property that the limits $f(x+), f(x-)$ exist. At first we recall that for a given positive integer $k$, the modulus of variation $v_{k}(h ; Y)$ of a bounded function $h \in \mathbb{R}^{[0, \infty)}$ on the interval $Y$ is defined as the upper bound of the set of all numbers $\sum_{j=1}^{k}\left|h\left(b_{j}\right)-h\left(a_{j}\right)\right|$ over all systems of $k$ non-overlapping intervals $\left(a_{j}, b_{j}\right)$ contained in $Y, j=\overline{1, k}$. If $k=0$, we take $v_{0}(h ; Y)=0$. We mention that some basic properties of this modulus can be found e.g. in [7].

We consider that the following requirements

$$
\left(H_{1}\right) \lim _{n \rightarrow \infty} a_{n}=0, \lim _{n \rightarrow \infty} n a_{n}=\infty \text {, }
$$

$\left(H_{2}\right) x>0$ such that the limits $f(x \pm)$ exist, are fulfilled.

Let $A$ be an arbitrary positive number for which $x \leq A$. Our hypotheses imply:

$$
\text { ( } \exists) n_{0},(\forall) n \geq n_{0}, \quad A<\frac{1}{\sqrt{a_{n}}}, \quad \frac{1}{3 n^{2} a_{n}^{2}} \leq \frac{1}{n a_{n}}
$$


and consequently ${ }_{n, 2}(x) \leq\left(x^{2}+x+1\right) d_{n}$ where

$$
d_{n}=\max \left\{\frac{1}{n}, A \sqrt{a_{n}}, \frac{1}{n a_{n}}\right\} .
$$

We also consider the mappings $g_{x}, s g n_{x}$ defined as follows

$$
g_{x}(t)=\left\{\begin{array}{cl}
f(t)-f(x-), & t \in[0, x) . \\
0, & t=x, \\
f(t)-f(x+), & t>x,
\end{array} \quad \text { and } \operatorname{sgn}_{x}(t)=\left\{\begin{array}{cl}
-1, & t \in[0, x), \\
0, & t=x, \\
1, & t>x .
\end{array}\right.\right.
$$

The estimate of $\left|\left(K_{n} f\right)(x)-f(x)\right|$ will be expressed in the terms of the modulus of variation of the function $g_{x}$.

Taking into account the above information and applying to our operators a result due to Grazyna Aniol, see [8; Theorem 2] we can state

Theorem 4. Let $K_{n}, n \in \mathbb{N}$, be defined by (2.1) such that the hypotheses $\left(H_{1}\right)$, $\left(H_{2}\right)$ are fulfilled and the function $f$ satisfies the growth condition $|f| \leq \psi$ with a positive continuous function $\psi$. We also suppose that for all $n \geq n_{0}$ and $t \geq 0$, $\left(K_{n} \psi^{2}\right)(t) \leq \varphi(t)$ holds, where $n_{0}$ is defined by (3.9). For every $n \geq n_{0}$ such that $d_{n} \leq 1 / 2$, we have

$$
\begin{gathered}
\left|\left(K_{n} f\right)(x)-\frac{f(x+)+f(x-)}{2}\right| \leq 2 P(x, A)\left\{\sum_{i=1}^{\mu_{n}-1} \frac{1}{i^{3}} v_{i}\left(g_{x} ; J_{x}\left(i A d_{n}\right)\right)+\right. \\
\left.+\frac{1}{\mu_{n}^{2}} v_{\mu_{n}}\left(g_{x} ; J_{x}(A)\right)\right\}+Q(x, A) d_{n}+\frac{1}{2}|f(x+)-f(x-)|\left|\left(K_{n} \operatorname{sgn}_{x}\right)(x)\right|,
\end{gathered}
$$

where $d_{n}$ is defined by (3.10), $\mu_{n}:=\left[1 / d_{n}\right], J_{x}(h):=[x-h, x+h] \cap[0, \infty)$ for $h>0$,

$$
P(x, A):=1+8\left(x^{2}+x+1\right) / A^{2}
$$

and

$$
Q(x, A):=A^{-1}\left(\left(x^{2}+x+1\right) \varphi(x)\right)^{1 / 2}+\frac{1}{2} A^{-2}\left(x^{2}+x+1\right) \psi(x) .
$$

Remarks. (i) If $f$ is continuous in $x$, then the last term in (3.11) vanishes.

(ii) If we consider the operators $K_{n}^{[\beta]}, n \in \mathbb{N}$, defined by (2.4), then we can take $d_{n}=\max \{1, A\} n^{-\lambda}$ where $\lambda:=\beta$ if $0<\beta<1 / 3$ and $\lambda:=(1-\beta) / 2$ if $1 / 3 \leq \beta<1$.

\section{REFERENCES}

[1] Balázs, C.: Approximation by Bernstein type rational functions, Acta Math. Acad. Sci. Hungar., 26(1-2), (1975), 123-134.

[2] Balázs, C. and Szabados, J.: Approximation by Bernstein type rational functions. II, Acta Math. Acad. Sci. Hungar., 40, (1982), 331-337. 
[3] Tотік, V.: Saturation for Bernstein type rational functions, Acta Math. Hung., 43(34), (1984), 219-250.

[4] Della Vecchia, B.: On some preservation and asymptotic relations of a rational operator, Facta Universitatis, 4, (1989), 57-62.

[5] Abel, U. and Della Vecchia, B.: Asymptotic approximation by the operators of $K$. Balázs and Szabados, Acta Sci. Math. (Szeged), 66, (2000), 137-145.

[6] Altomare, F. and Campiti, M.: Korovkin-Type Approximation Theory and its Applications, De Gruyter Series Studies in Mathematics, Vol.17, Walter de Gruyter, Berlin - New York, 1994.

[7] Chanturiya, Z.A.: Modulus of variation of function and its application in the theory of Fourier series, Dokl. Akad. Nauk SSSR, 214, (1974), 63-66 (in Russian).

[8] AnIOL, G.: On the rate of pointwise convergence of the Kantorovich-type operators, Fasciculi Mathematici, 29, (1999), 5-15. 